\title{
Exploiting Phase Diversity for CDMA2000 1X Smart Antenna Base Stations
}

\author{
Seongdo Kim \\ Advanced ReD Team, Ace Technology, Seoul 137-130, Korea \\ Email: ksd544@acetech.co.kr

\section{Seungheon Hyeon} \\ School of Electrical and Computer Engineering, Hanyang University, Seoul 133-791, Korea \\ Email:hsheon@dsplab.hanyang.ac.kr \\ Seungwon Choi \\ School of Electrical and Computer Engineering, Hanyang University, Seoul 133-791, Korea \\ Email: choi@ieee.org
}

Received 25 June 2003; Revised 4 March 2004

A performance analysis of an access channel decoder is presented which exploits a diversity gain due to the independent magnitude of received signals energy at each of the antenna elements of a smart-antenna base-station transceiver subsystem (BTS) operating in CDMA2000 1X signal environment. The objective is to enhance the data retrieval at cellsite during the access period, for which the optimal weight vector of the smart antenna BTS is not available. It is shown in this paper that the access channel decoder proposed in this paper outperforms the conventional one, which is based on a single antenna channel in terms of detection probability of access probe, access channel failure probability, and Walsh-code demodulation performance.

Keywords and phrases: phase diversity, access channel, searching, Walsh-code demodulation, CDMA2000 1X.

\section{INTRODUCTION}

As the demand of mobile communications increases rapidly, the 3G mobile communication system must provide various contents in as high as possible data rate compared to the conventional $2 \mathrm{G}$ systems [1]. In order to provide the various contents to the increased number of users, it is necessary to secure an extremely accurate detection of synchronization information between base-station transceiver subsystem (BTS) and each of the subscribers together with a good demodulation technique [2]. For improving the quality of communication services capable of providing the various contents, the mobile communication network based on a single antenna BTS must increase the number of base stations. However, to increase the number of base stations is very costly and it also causes the cell planning to be very complicated because of the frequent handoff [3].

Smart antenna technology $[4,5]$ has been considered as being a solution to increase the communication capacity and improve the communication quality $[6,7]$ as well without too much investment required for increasing the number of base stations. In order for the smart antenna system to work as desired, however, the weight vector should be provided in such a way that a nice-shaped beam pattern is generated in accordance with the directions of desired and/or undesired signal sources [8].

It is noteworthy that the data format of the access channel in CDMA2000 1X system is the same as that employed in the current IS-95 CDMA system. It particularly means that access channel consists of the preamble, data, and cyclic redundancy code (CRC) with the data rate being $4.8 \mathrm{kbps}$. As the access channel is not accompanied with pilot channel, the contents of the access channel should be decoded through a noncoherent detection $[9,10,11]$. For that reason, during the access state, which exists before a traffic channel is set up between BTS and a given subscriber such that an optimal weight vector has not yet been computed according to the received data at the BTS, the advantages of smart antenna, which is available due to the nice-shaped beam pattern, cannot be provided. In this paper, we apply the principle of phase diversity technology [12] to the access channel decoder in order to enhance the data retrieval during the access state. The phase diversity can be obtained in any antenna array system which uses the envelope detection procedure regardless of antenna spacing due to the fact that the 
energy of received signal at each antenna element consists of many signal components transmitted from a large number of users with all statistically independent arrival angles. Consequently, the energy at each antenna element becomes statistically independent as the number of users increases in a given CDMA signal environment. The gain of phase diversity can be exploited in the access channel decoder, of which the function is basically to compute the correlation energy of received signal with each of the Walsh codes by simply summing up the correlation energies calculated at each of the antenna elements.

The superiority of the proposed access channel decoder exploiting the phase diversity has been confirmed in terms of searching capability, Walsh demodulation, access failure probability, and so forth. In this paper, the access failure has been found by checking the CRC that is contained in the access probe.

This paper is composed as follows. Section 2 summarizes the concept of the phase diversity. Section 3 introduces the structure of access channel decoder for a hardware implementation. Section 4 presents the performance analysis of the proposed access channel decoder in comparison to the conventional one. Section 5 presents the concluding remarks.

\section{PHASE DIVERSITY}

Phase diversity technology is based on a fact that the magnitude of received signal energy at each antenna element of a smart antenna system is independent of each other because the phase of every component, which is determined by the arrival angle and carrier phase delay associated with the corresponding mobile terminal, is statistically independent of each other. It may sound contradictory to another fact that the received signal at each antenna element is coherent to each other, that is, the magnitude of received signal is the same at every antenna element and only the phase varies when the angle spread is not too wide and the antenna spacing is not far greater than a half wavelength. What has to be carefully considered in the discussion of the phase diversity technology is that the received signal at each antenna element is composed of plural signal components, each of which is transmitted from a corresponding mobile terminal. Each of the received signal components at each antenna element is fully coherent to each other such that the magnitude is exactly the same at every antenna element and the phase difference between adjacent elements is $\pi \sin (\theta)$ when the antenna spacing is a half wavelength where $\theta$ is the arrival angle measured from the broad side of the array. However, due to the large number of transmitting mobile terminals, the magnitude of the received signal which consists of the large number of the signal components is independent at every antenna element. The independency of the received signal energy can be clarified through the equations shown in this section.

The phase difference between adjacent antenna elements due to a single signal component, say, a signal transmitted from $l$ th subscriber, is a function of incident angle $\theta_{l}$ and antenna spacing $d$, that is, $\varphi\left(\theta_{l}, d\right)$. For simplicity but without loss of generality, we assume that the first antenna element is the reference antenna. Then, holding back the terms related to the multipath and angle spread until we quote Section 4, after the frequency down-conversion, the in-phase and quadrature component of the received signal at the $n$th element can be respectively written as follows:

$$
\begin{aligned}
\hat{I}_{n}(t) & =\sum_{m=1}^{M} \hat{S}_{m}(t) \cos \left[\phi_{m}+(n-1) \varphi\left(\theta_{m}, d\right)\right], \\
\hat{Q}_{n}(t) & =\sum_{m=1}^{M} \hat{S}_{m}(t) \sin \left[\phi_{m}+(n-1) \varphi\left(\theta_{m}, d\right)\right],
\end{aligned}
$$

where the subscripts $n$ and $m$ are the indices for denoting the antenna element and signal source, that is, the transmitting subscriber, respectively, $\hat{S}_{m}(t)$ is the magnitude of the received signal, $M$ is the total number of signal components impinging upon the antenna element, and $\phi_{m}$ is the carrier phase delay. The noise term has been deleted for ease of explanation. Assuming that the signal transmitted from the $l$ th subscriber is the desired one, after the dispreading procedure with the PN code assigned to the $l$ th subscriber, say, $p_{l}(t)$, the in-phase and Quadrature component of the received signal at the $n$th element can be written as follows:

$$
\begin{aligned}
I_{n}(t)= & S_{l}(t) \cos \left[\phi_{l}+(n-1) \varphi\left(\theta_{l}, d\right)\right] \\
& +\sum_{m=1, m \neq l}^{M} S_{m}(t) \cos \left[\phi_{m}+(n-1) \varphi\left(\theta_{m}, d\right)\right], \\
Q_{n}(t)= & S_{l}(t) \sin \left[\phi_{l}+(n-1) \varphi\left(\theta_{l}, d\right)\right] \\
& +\sum_{m=1, m \neq l}^{M} S_{m}(t) \sin \left[\phi_{m}+(n-1) \varphi\left(\theta_{m}, d\right)\right],
\end{aligned}
$$

where $I_{n}(t)=\int_{T} \hat{I}_{n}(t) p_{l}(t) d t, Q_{n}(t)=\int_{T} \hat{Q}_{n}(t) p_{l}(t) d t$, and $S_{m}(t)=\int_{T} \hat{S}_{m}(t) p_{l}(t) d t$ with the integral period $T$ being determined by the processing gain. Note that the first terms in the right-hand side of (2) are the desired ones while the last terms are the interfering ones. The key part of the phase diversity is that the signal at each antenna element is independent of each other because the signal at each antenna element consists of a large number of signals transmitted from randomly located large number of mobile terminals. Once again, this is because the interfering terms consist of $M-1$ terms of which the incident angles are all independent such that the magnitude of the received signal at each element, which is determined by a vector sum of $M$ signal components, must be determined in a random fashion.

In Walsh-code demodulator in access channel decoder, as there are 64 Walsh codes in CDMA2000 1X system, each symbol shown in (2) should be correlated with each of the 64 Walsh codes at each antenna element to produce the decision variables of Walsh demodulator. Then, the decision variables for $k=1,2, \ldots, 64$ at the $n$th antenna element, which is obtained by correlating the symbol with each of the 64 Walsh 
codes, can be written as follows:

$$
\begin{aligned}
I_{n, k}(t)= & S_{l, k}(t) \cos \left[\phi_{l}+(n-1) \varphi\left(\theta_{l}, d\right)\right] \\
& +\sum_{m=1, m \neq l}^{M} S_{m, k}(t) \cos \left[\phi_{m}+(n-1) \varphi\left(\theta_{m}, d\right)\right], \\
Q_{n, k}(t)= & S_{l, k}(t) \sin \left[\phi_{l}+(n-1) \varphi\left(\theta_{l}, d\right)\right] \\
& +\sum_{m=1, m \neq l}^{M} S_{m, k}(t) \sin \left[\phi_{m}+(n-1) \varphi\left(\theta_{m}, d\right)\right],
\end{aligned}
$$

where the subscript $k$ is the Walsh index and the $k$ th decision variable is obtained as $I_{n, k}(t)=\int_{T_{W}} I_{n}(t) W_{k}(t) d t$, $Q_{n, k}(t)=\int_{T_{W}} Q_{n}(t) W_{k}(t) d t$, and $S_{m, k}(t)=\int_{T_{W}} S_{m}(t) W_{k}(t) d t$ for $k=1,2, \ldots, 64$ with the integral period $T_{W}$ being determined by the length of the Walsh code. Since the interfering terms in (3) can be approximated to Gaussian as the number of signal components is sufficiently large, (3) can be rewritten as follows:

$$
\begin{aligned}
I_{n, k}(t) & =G\left[S_{l, k}(t) \cos \left(\Theta_{n, l}\right), \sigma^{2}\right], \\
Q_{n, k}(t) & =G\left[S_{l, k}(t) \sin \left(\Theta_{n, l}\right), \sigma^{2}\right],
\end{aligned}
$$

where $G\left[\mu, \sigma^{2}\right]$ denotes a Gaussian random variable with mean $\mu$ and variance $\sigma^{2}$, where the variance is determined by the sum of the interferers' power measured at the receiver, and $\Theta_{n, l}=\phi_{l}+(n-1) \varphi\left(\theta_{l}, d\right)$.

What is claimed in the phase diversity technology is that the decision variable should be computed as a sum of all the values obtained at every antenna channel. Thus, the decision variable $Z_{k}(t)$ for estimating the Walsh number in the Walsh demodulator is

$$
Z_{k}(t)=\sum_{n=1}^{N}\left[I_{n, k}^{2}(t)+Q_{n, k}^{2}(t)\right] \quad \text { for } k=1,2, \ldots, 64 .
$$

As $I_{n, k}(t)$ and $Q_{n, k}(t)$ are Gaussian random variables as discussed above, the decision variable $Z_{k}(t)$ is a noncentric chi-squared random variable with the degree of freedom being $2 N$. The probability density function of $Z_{k}(t)$ can be written as follows [13]:

$$
\begin{array}{ll}
P_{Z}(\alpha) & \text { for } \alpha \geq 0, \\
=\frac{\left(\alpha / \sigma^{2} b\right)^{(N-1) / 2}}{2 \sigma^{2}} e^{-(1 / 2)\left(b+\alpha / \sigma^{2}\right)} I_{N-1}\left(\sqrt{\frac{b \alpha}{\sigma^{2}}}\right) & \text { for } \alpha<0,
\end{array}
$$

where $I_{N-1}(\bullet)$ is a modified Bessel function of the first kind with order $N-1$ and the noncentric parameter $b$ is $N\left(S_{l, k}(t)^{2} / \sigma^{2}\right)$. Note that when the magnitude of the desired signal is zero, the probability density function of $Z_{k}(t)$ becomes a centric chi-squared random variable. In this case, the probability density function of $Z_{k}(t)$ can be written as follows:

$$
p_{Z}(\alpha)=\frac{\alpha^{(N-1)}}{\left(\sigma^{2}\right)^{N} 2^{N} \Gamma(N)} e^{-\alpha / 2 \sigma^{2}}
$$

where $\Gamma(\bullet)$ is the Gamma function.
The average and variance of the centric chi-squared random variable are $2 N \sigma^{2}$ and $4 N \sigma^{4}$, respectively, and those for the noncentric chi-squared random variable are $N\left(2 \sigma^{2}+\right.$ $\left.S_{l, k}(t)^{2}\right)$ and $4 N \sigma^{2}\left(\sigma^{2}+S_{l, k}(t)^{2}\right)$, respectively. Note that both the mean and variance increase linearly as the number of antenna elements $N$ increases. This suggests that the performance of the envelope detection, that is, $Z_{k}(t)=$ $\sum_{n=1}^{N}\left[I_{n, k}^{2}(t)+Q_{n, k}^{2}(t)\right]$, improves linearly as the number of antenna elements increases. Note that there would be no gain at all if the variance increases in proportion to $N^{2}$ as the mean increases in proportion to $N$. Consequently, the phase diversity technique, with the detection variables being computed as suggested in (5), increases the signal-to-interference ratio (SIR) by nearly $N$ times where $N$ is the number of antenna elements in the array system. Note that there is no weight computation involved in the phase diversity technique.

\section{ACCESS CHANNEL DECODER}

Figure 1 illustrates a block diagram of the access channel modulator operating in CDMA2000 1X mobile communication system. As the terminal may access the BTS at any moment, the BTS in CDMA2000 1X system receives the access information based on a noncoherent detection. Note that, as shown in Figure 1, the information in the access channel is transmitted in a low data rate, that is, $4.8 \mathrm{kbps}$. It can also be observed in Figure 1 that the access channel modulator includes the 64-ary Walsh modulation and the offset quadrature phase shift keying (OQPSK) modulation [14]. It is also noteworthy that the OQPSK modulation in the access channel modulator can provide a diversity gain because it adopts two BPSK modulations for the I-channel and Q-channel, respectively, of a given signal.

The access channel decoder implemented in our smart antenna BTS consists of a searcher, OQPSK demodulator, Walsh demodulator, Viterbi decoder, CRC checker, and so forth, in such a way that it can demodulate the access channel data modulated through the procedure shown in Figure 1. Among the blocks in the access channel decoder, the searcher and Walsh demodulator employs the proposed phase diversity technology of which the detailed application method and hardware structures are introduced in Sections 3.1 and 3.2.

\subsection{Searcher}

The searcher in the access channel decoder performs PN code acquisition for retrieving the access channel information at cellsite, using the preambles given at the beginning part of access probe [15]. As described earlier, in order to exploit the phase diversity gain, the correlation energies obtained at each of the antenna channels are summed up each time to produce the detection variable, with which the searcher detects the peak correlation energy to estimate the propagation delay of the target subscriber. Figure 2 illustrates the hardware structure of the access searcher implemented in our smart antenna system operating in CDMA2000 1X system. Note that the correlation energies computed at each of the antenna channels are summed up to form the detection variable for determining whether or not the current time lag corresponds 

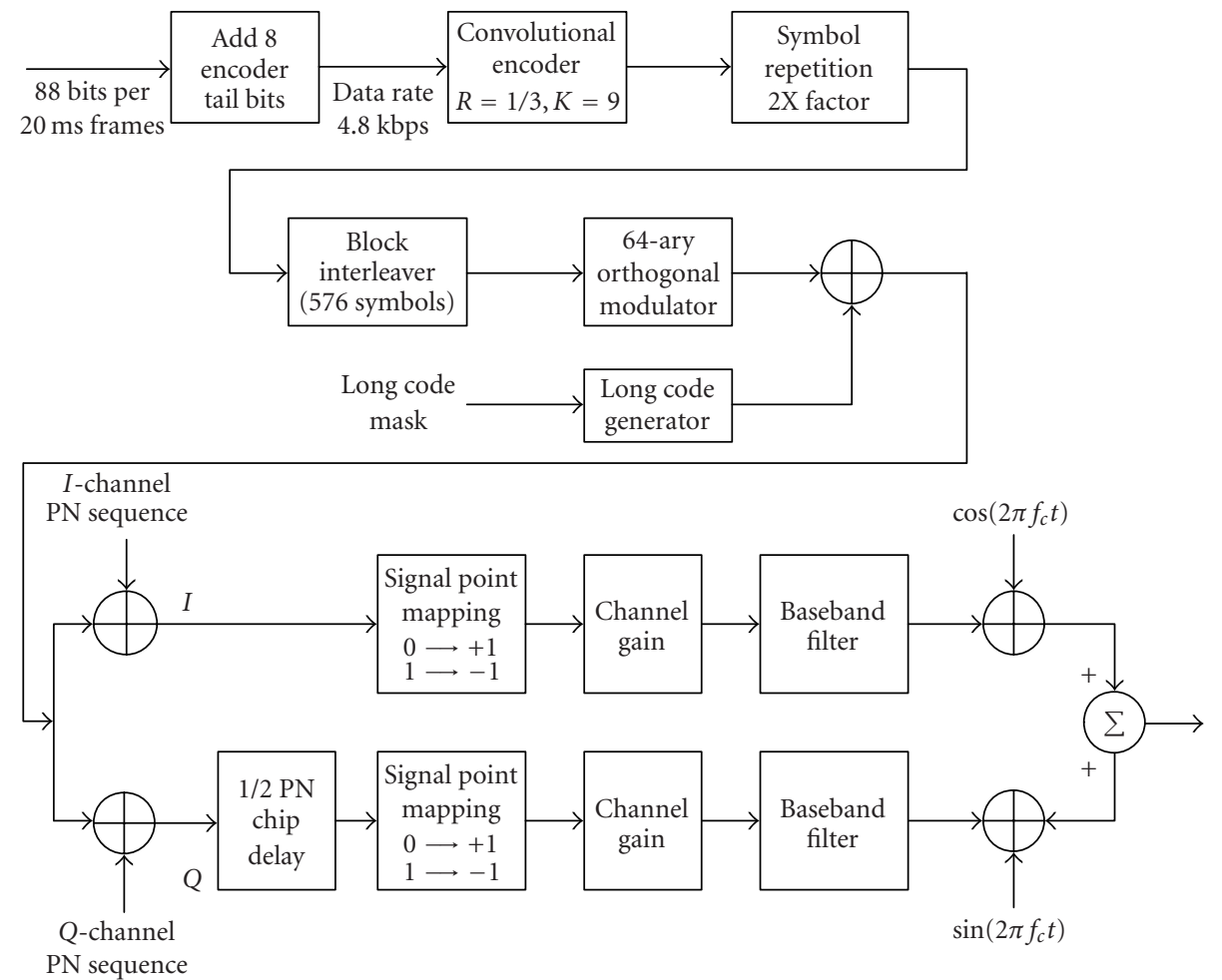

FIgURE 1: Structure of access channel modulator of CDMA2000 1X system.

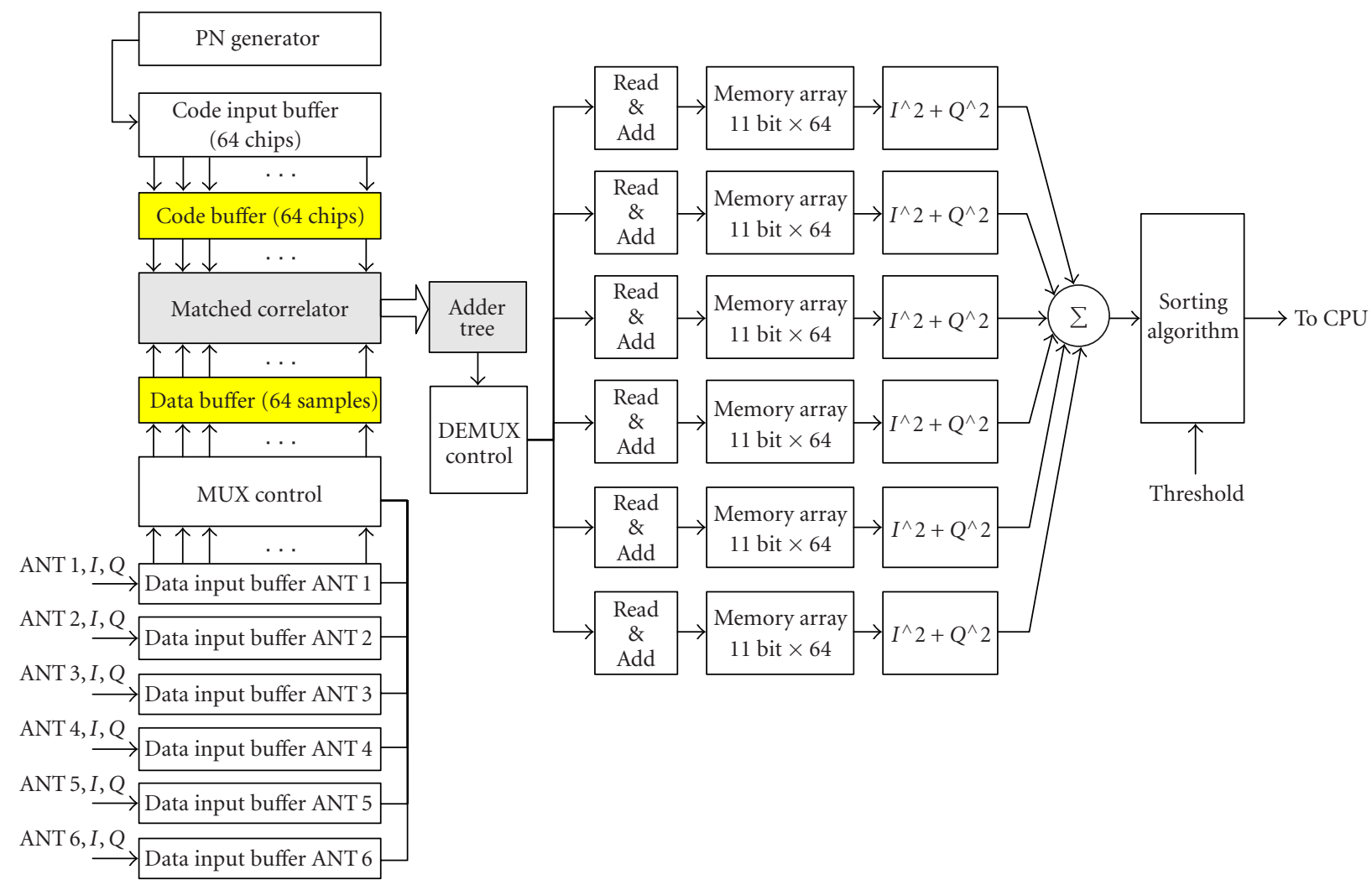

FigURE 2: Structure of the proposed searcher. 
to the peak correlation value. In our smart antenna BTS, 6 antenna channels are activated for reverse link, that is, $N=6$.

As shown in Figure 2, the $I$ and $Q$ components of the received signal obtained after the frequency-down and analog-to-digital conversion at each antenna channel, that is, $\left\{\mathrm{ANT}_{i} I, Q\right.$ for $\left.I=1,2, \ldots, N\right\}$, respectively, are fed to the corresponding data input buffer.

The searching function, that is, $\mathrm{PN}$ code acquisition, is performed after analog-to-digital conversion of frequency down-converted baseband signal received at each of the antenna channels. The detailed procedure of computing the correlation energy in the hardware implementation of our access channel searcher is as follows. First, the correlation between the received data and PN code of the desired subscriber is performed for the period of 1/4 PCG (power control group), which is of 384-chip duration, with the time lag of correlation being shifted by $1 / 2$ chip at a time. At each antenna channel, the correlation energy is obtained by averaging the correlation results for the period of 7 PCG. Consequently, the total period for obtaining the correlation energy at each antenna channel is for $7 \times 4 \times 384$ chips. As for the window size, that is, the length of the time interval for which the peak correlation energy is searched, it has been set to 30-chip duration, which means the correlation energy is computed 60 times because the time lag for computing the correlation is jumping by $1 / 2$ chip each time the correlation is computed as mentioned above. Summarizing the above, the searching energy is computed for each time lag $i$ by

$$
\begin{aligned}
& Z_{\text {search }}\left(\frac{i}{2}\right) \\
& =\sum_{j=1}^{N}\left\{\begin{array}{l}
\left\{\sum_{k=0}^{28} \sum_{n=1}^{384} x_{j}^{I}[n+384 \times k]\right. \\
\left.\cdot \mathrm{PN}^{I}\left[n+384 \times k-\frac{i}{2}\right]\right\}^{2} \\
+\left\{\sum_{k=0}^{28} \sum_{n=1}^{384} x_{j}^{Q}[n+384 \times k]\right. \\
\left.\cdot \mathrm{PN}^{Q}\left[\left(n-\frac{1}{2}\right)+384 \times k-\frac{i}{2}\right]\right\}^{2} \\
\text { for } i=0,1,2, \ldots, 59,
\end{array}\right\}
\end{aligned}
$$

where $Z_{\text {search }}(t)$ is the searching energy to be computed, $x_{j}^{I}(t)$ and $x_{j}^{Q}(t)$ are, respectively, the in-phase and quadrature component of the received data at $j$ th antenna element, and $\mathrm{PN}^{I}(t)$ and $\mathrm{PN}^{Q}(t)$ denote the $\mathrm{PN}$ code of the desired subscriber assigned to In-phase and Quadrature component, respectively, in CDMA2000 1X system. Note that the time index includes $(i / 2)$ term because the time lag for computing the correlation energy is shifted by $1 / 2$-chip duration each time as described previously. It should also be observed that the received data $x_{j}^{I}(t)$ and $x_{j}^{Q}(t)$ consist of many signal components transmitted from all the subscribers operating in a given cell through the traffic as well as access channels. In the access searcher operating in accordance with (8), the phase diversity can be exploited because the correlation energy is obtained through the summation of all the correlation results at each of the antenna channels. The objective of the searcher in the access channel decoder is to find the time index $(i / 2)$ for which the peak value of the correlation energy is given as a result of computing (8) for every $i$. The problem of setting the threshold value to determine whether or not each of the correlation values corresponds to a peak is not included in the scope of this paper.

\subsection{Walsh demodulator}

The objective of the Walsh demodulator is to find the Walsh number that corresponds to the information of 6-bit word transmitted from the desired subscriber. So, the question to be answered in the Walsh demodulator is "Which one of $\left\{W_{0}, W_{1}, W_{2}, \ldots, W_{63}\right\}$ has been transmitted from the target subscriber?" Figure 3 illustrates the Walsh demodulator implemented in our smart antenna BTS [16] operating in the reverse link of CDMA2000 1X signal environment that can fully exploit the gain of phase diversity [17]. At each antenna channel, the received signal is first mixed with $\cos \omega_{1} t$ and $\sin \omega_{1} t$ to produce the baseband in-phase and quadrature component $r_{I, n}(t)$ and $r_{Q, n}(t)$, respectively, for $n=1,2, \ldots, N$, where $\omega_{1}$ is the carrier frequency with $N$ being the number of antenna elements in the smart antenna system. For ease of explanation, A-to-D converter is omitted in Figure 3. Then, the received data are descrambled with the long and short PN code, that is, $c(t)$, and $p_{I}(t)$ and $p_{Q}(t)$ for in-phase and quadrature, respectively, in Figure 3, assigned to the desired subscriber. Note that the descrambling procedure is performed using the timing information, that is, $(i / 2)$ as shown in (8), given from the access searcher. Then, the descrambled received data are fed to the input ports of the Walsh demodulator at each antenna channel to be correlated with each of the 64 Walsh words. In Figure 3, the correlation of the received data with each of the Walsh words is denoted as $\left\langle\bullet, W_{k}(t)\right\rangle$, which is performed by $\int_{T_{W}} \bullet W_{k}(t) d t$ with the integration period $T_{W}$ being the Walsh word length as mentioned in Section 2. Note that in order to provide the phase diversity gain mentioned earlier, the decision variable $Z_{k}$ for $k=1,2, \ldots, 64$ is obtained through the summation procedure of correlation values computed at each of the antenna channels in Figure 3 [3]. As the objective of the Walsh demodulator is irrelevant to the time index, the time index in the decision variable $Z_{k}(t)$ as shown in (5) is omitted in this section.

Figure 4 is the photograph of a channel card implemented in our smart antenna BTS [17]. Each channel card installed in a given smart antenna BTS includes all the necessary modules for modulating or demodulating the signal to be transmitted or received to or from the corresponding subscriber. The access channel searcher and the Walsh demodulator discussed in this section are included in the demodulation part of the channel card. As shown in Figure 4, the demodulator has been implemented with 5 of 1 milliongate FPGAs (field programmable gate array)_Altera's APEX EP20K1000EBC652. In fact, the demodulation part of the channel card consists of the access channel searcher, Walsh 


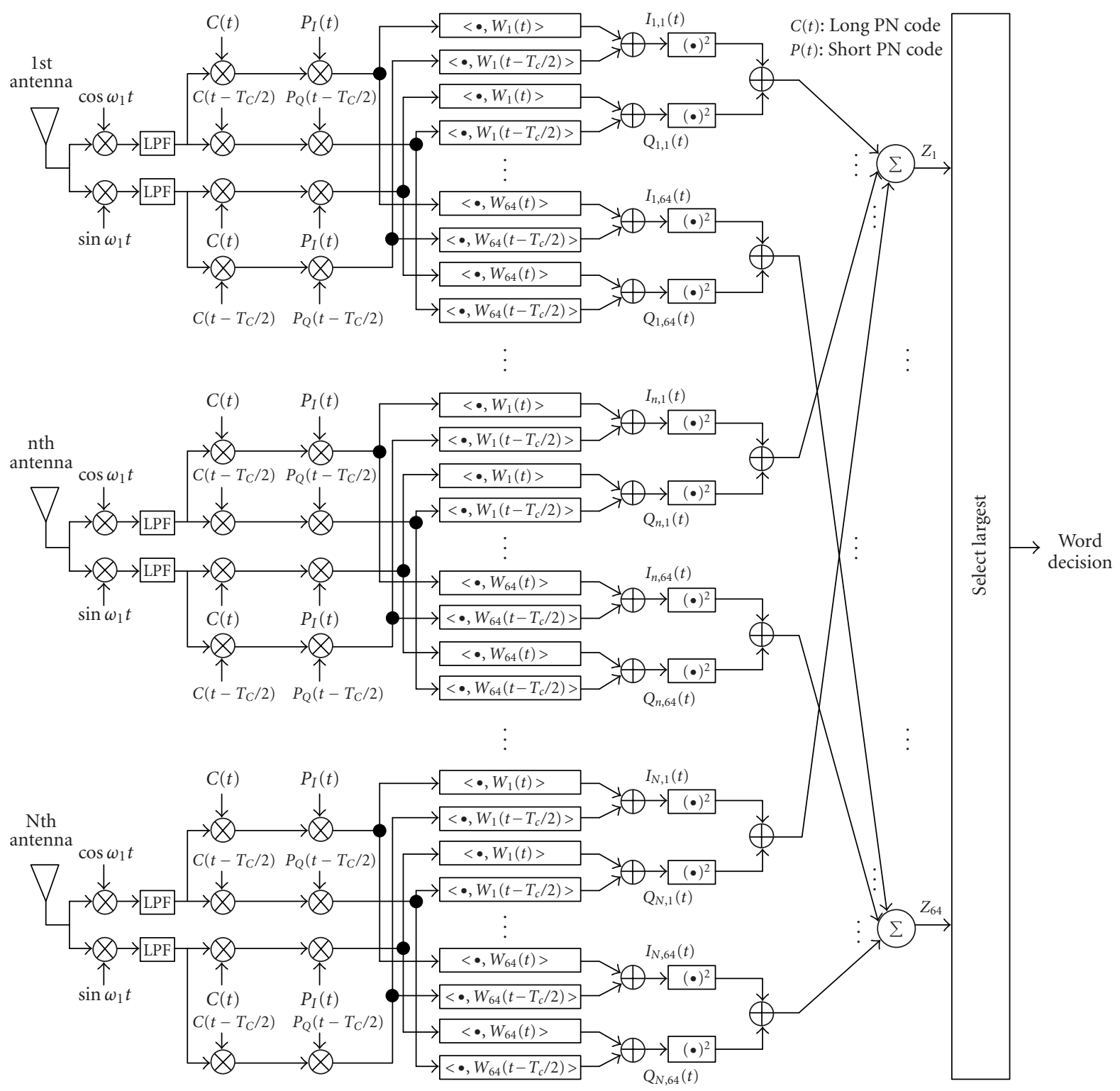

FIGURE 3: Walsh demodulator providing the phase diversity in smart antenna base station.

demodulator, both of which are discussed in this section as main topics of this paper, traffic channel demodulator for dispreading the received traffic data, demodulator controller that has been implemented with a digital signal processing (DSP)-TMS320C6203, and channel card controller that has been implemented with MPC860. All these modules for the demodulation have been implemented in the 5 FPGAs shown in Figure 4. The demodulator shown in Figure 4 provides 4 fingers to each user for the RAKE reception [18]. The received data through the access channel allocated to each of the 4 fingers by the access searcher are demodulated in the Walsh demodulator implemented with the FPGAs. The demodulator controller demodulates the access probe from the retrieved 6-bit words which are obtained as a result of the Walsh demodulation. The state of the desired subscriber can also be monitored from these 6-bit words information because the CRC of the desired subscriber is included in the access probe. The CRC information are transferred to the channel card controller. It is also the channel card controller that performs the interface between physical layer and higher layer as well as the call processing using the message retrieved from the access probe.

\section{PERFORMANCE ANALYSIS}

\subsection{Signal modeling and experimental environment}

In this subsection, the performance analysis of the proposed access channel decoder is presented through various computer simulations and experimental results. The received baseband signal at the $n$th antenna element, assuming the 


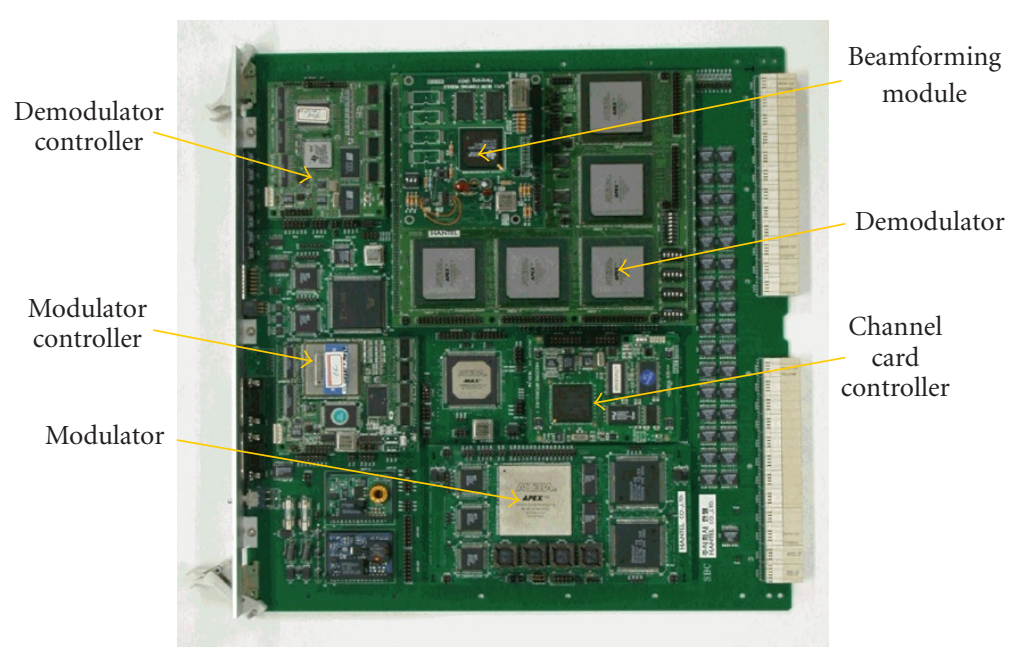

FIGURE 4: Channel card for smart antenna system.

$n_{0}$ th element is reference antenna element, can be written as follows $[19,20]$ :

$$
\begin{aligned}
x_{n}(t)=\sum_{m=1}^{M} \sum_{k=1}^{K_{m}} & \left(\sum_{q=1}^{L_{k}} s_{m, k}(t) e^{j 2 \pi\left(f_{d} \cos \varphi_{m, k, q} t-f_{c} \tau_{m, k, q}\right)}\right) \\
& \times e^{-j\left(n-n_{0}\right) \pi \sin \theta_{m, k, q}}+n(t),
\end{aligned}
$$

where the indices $m, k$, and $q$ are used to denote the subscriber, propagation path, and scattered components, respectively, $s_{m, k}(t)$ is the received signal from the $m$ th subscriber through the $k$ th path, $f_{d}$ is Doppler shift, $\varphi$ is the moving direction of the desired subscriber measured from the broad side of the array antenna, $f_{c}$ is the carrier frequency, $\tau$ is the propagation delay of the signal, $\theta$ is the arrival angle of the received signal, and $n(t)$ is a zero-mean Gaussian random quantity determined by the noise. Note that the signal model shown in (9) includes the angular spread. Note that $M, K_{m}$, and $L_{k}$ are the number of subscribers, multipaths, and scattered components, respectively.

It has been assumed in our simulations that the propagation delay in a cluster are all the same such that $\tau_{m, k, q} \cong \tau_{m, k}$ [19]. The magnitude of each of the multipaths has been determined in accordance with the 6-finger model given in 3GPP2 recommendation as follows: $0.6369: 0.5742$ : $0.3623: 0.2536: 0.2595: 0.0407$, which results in the power ratio of $0.4056: 0.3297: 0.1313: 0.0643: 0.0673: 0.0017$. Note that for the retrieval of access channel data, which is the main issue of this paper, the largest 4 fingers out of the 6 , of which the instantaneous magnitude varies at every sampling time due to the fading, are taken because the number of fingers in our receiver is 4 as mentioned earlier.

\subsection{Performance of the searcher}

In the access state, during which the pilot signals are not available, the searching, that is, PN code acquisition, is performed using the preamble given at the access probe. In

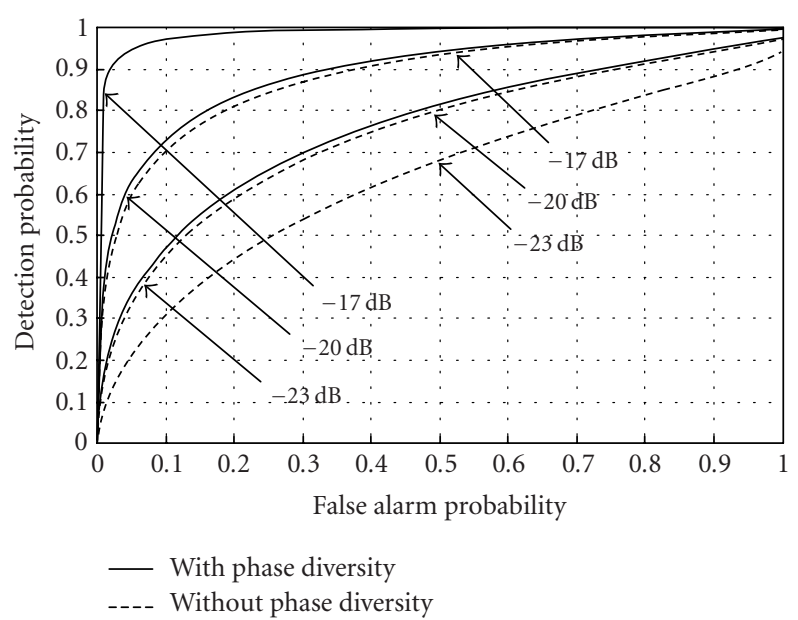

FIGURE 5: False alarm probability versus detection probability in AWGN environment.

our smart antenna BTS, the access probe is composed of 5 -frame preamble and 4-frame message. The integration period for the searcher has been set to 384-chip duration ( $312.5 \mu$ second), which is $1 / 4$ PCG. As the phase shift of the received signal is about $9^{\circ}$ for $f_{d}=80 \mathrm{~Hz}$ for the integration period of $312.5 \mu$ second, the correlation energy in the searcher is obtained by averaging the correlation values for the period of 7 PCG for higher accuracy as stated in Section 3.

Figures 5 and 6 illustrate the performance of the access searcher in terms of false alarm probability and detection probability in additive white Gaussian noise (AWGN) and fading channel, respectively. The numbers given inside the parenthesis in Figures 5 and 6 denote the signal-tointerference-plus-noise ratio (SINR), being $-17 \mathrm{~dB},-20 \mathrm{~dB}$, and $-23 \mathrm{~dB}$. It has been found in our extensive computer simulations that the searcher designed by the proposed 


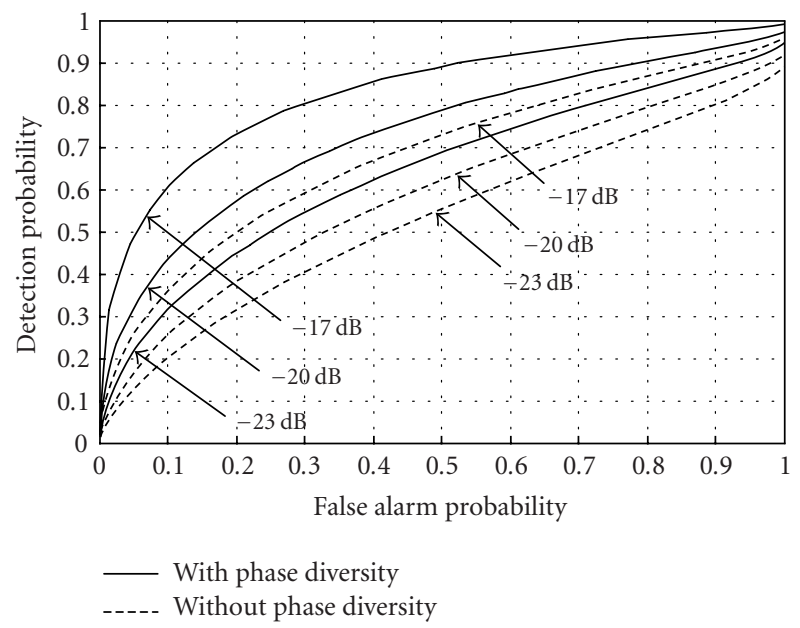

FIGURE 6: False alarm probability versus detection probability in the fading environment.

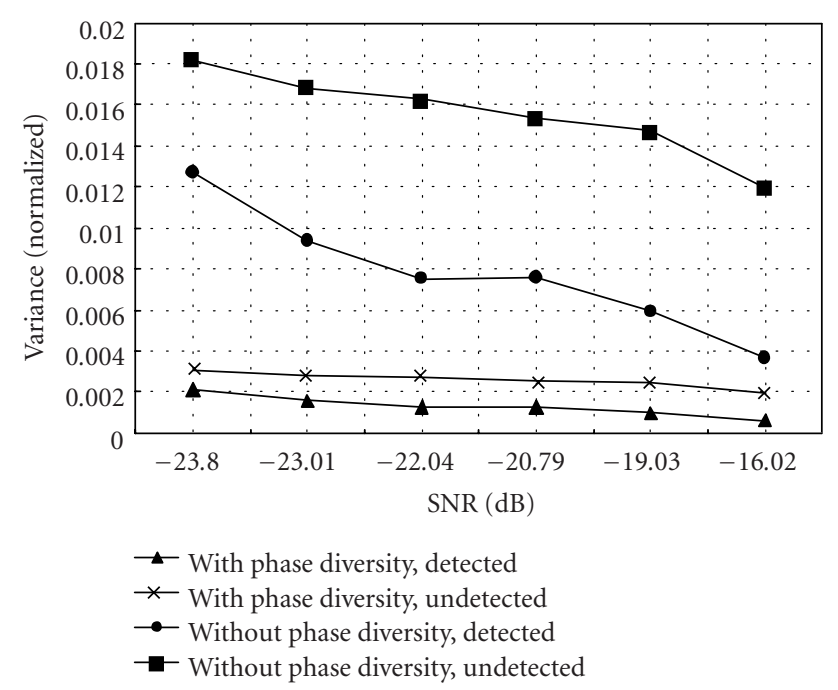

FIgURE 7: Variance of searching energy.

technique that exploits the phase diversity provides for about $3.3 \mathrm{~dB}$ gain in the SINR for AWGN channel. The gain in the fading environments has been found to be about $5.5 \mathrm{~dB}$.

The performance improvement provided by the proposed searcher can also be found in the distribution of the variance of the detection variable, that is, $E[\{Z(i / 2)-$ $\left.E[Z(i / 2)]\}^{2}\right]$, where $E[\bullet]$ denotes the expectation of $\bullet$ and $Z(i / 2)$ is the detection variable, that is, correlation energy, as defined in (8). Figure 7 illustrates the variance of the normalized detection variable. Note that the labeling "detected" or "undetected" means that the time lag of the detection variable, that is, $(i / 2)$, is matched or mismatched with the actual propagation delay of the signal, respectively. In computing the variance, the correlation energy itself has been normalized with the average correlation energy, that is, $E\left[Z\left(i_{0} / 2\right)\right]$, obtained in the detected case. It should be ob-

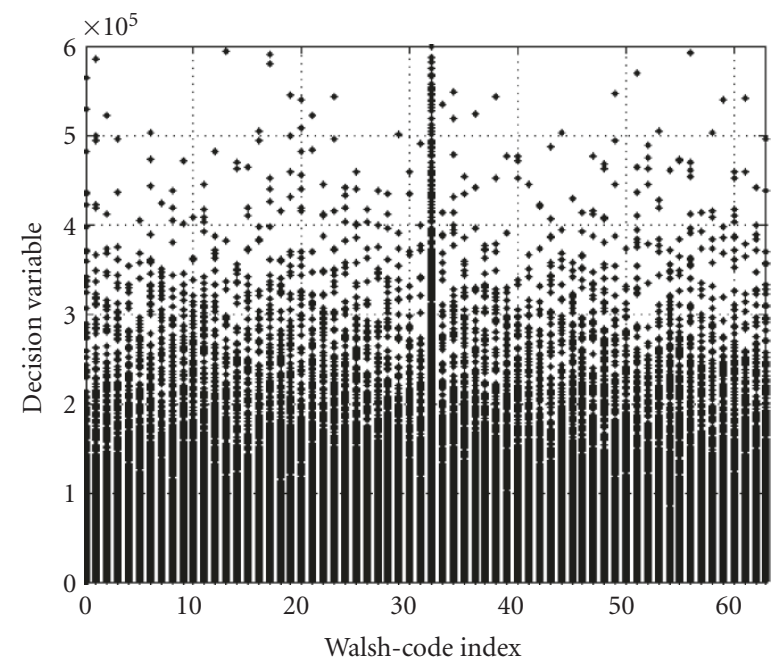

Figure 8: Decision variable of the Walsh demodulator for single antenna system.

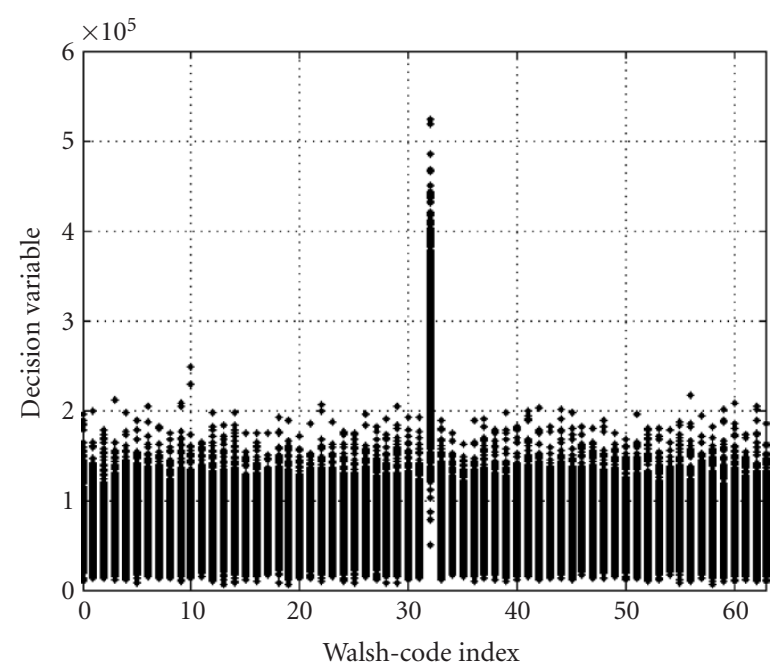

Figure 9: Decision variable of the Walsh demodulator for array antenna system.

served in Figure 7 that the variance in both the detected and undetected cases is significantly reduced, which means the detection capability is enhanced and the false alarm is suppressed as much as the variance is reduced. Recall that the variance of the correlation energy is actually an uncertainty in the detection procedure of the searcher. It has been found in our extensive simulations that the variance is reduced nearly by $1 / N$, that is, $1 / 6$ in our smart antenna BTS. This result could be predicted from the discussions of Section 2 .

\subsection{Performance of the Walsh demodulator}

The performance of the Walsh demodulator is presented in terms of the distribution of the detection variable $Z_{k}$ and the improvement in $E_{b} / N_{0}$. Figures 8 and 9 illustrate the distribution of the decision variable $Z_{k}$ in an AWGN channel of $\mathrm{SNR}=-24 \mathrm{~dB}$. Each of the 64 Walsh words is correlated with 


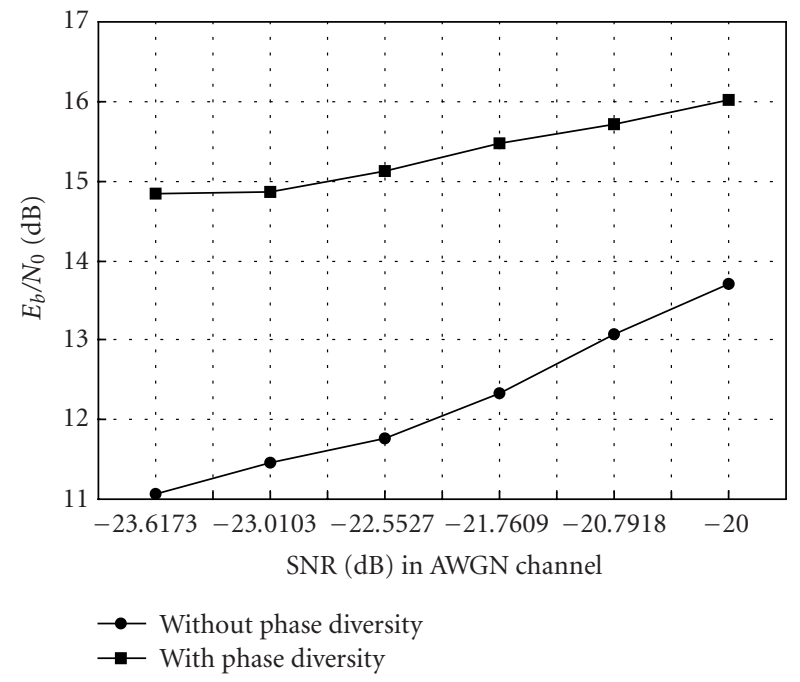

FIgURE 10: $E_{b} / N_{0}$ enhancement in the Walsh demodulator.

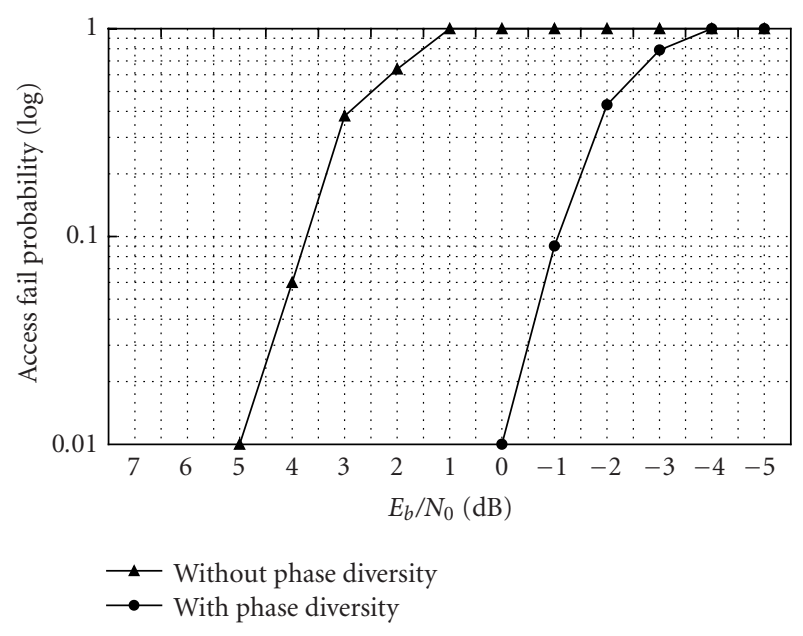

FIGURE 11: Access fail probability in AWGN environment.

received signal of 64-chip length when a test signal corresponding to $W_{32}$ is assumed to be transmitted from a mobile terminal. From Figures 8 and 9, it can be observed that the decision variable $Z_{32}$ provided from the proposed Walsh demodulator employing the phase diversity technology is significantly distinguished from the other correlation results, that is, $Z_{i, i \neq 32}$, because the variance in computing each of the correlation results in the proposed system is reduced almost proportionally to the number of antenna elements, that is, 6 in our smart antenna BTS. Consequently, the performance of the Walsh demodulator is improved by adopting the array system with phase diversity.

Figure 10 illustrates an improvement of the proposed Walsh demodulator in terms of $E_{b} / N_{0}$. The value for $E_{b} / N_{0}$ has been obtained from the ratio between $E\left[Z_{32}\right]$ and the standard deviation in computing the average value of the decision variable. As shown in Figure 10, $E_{b} / N_{0}$ in the Walsh demodulator is increased by about $2.5 \sim 4 \mathrm{~dB}$.

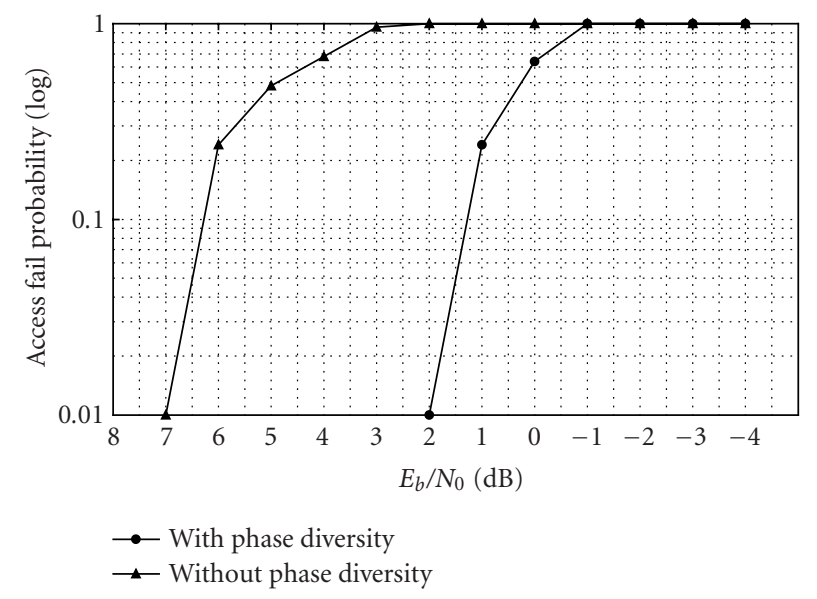

FIgure 12: Access fail probability in the fading environment.

TABle 1: Measured $E_{b} / N_{0}$ at access channel decoder output.

\begin{tabular}{c|cc}
\hline \multirow{2}{*}{ Channel $E_{b} / N_{0}(\mathrm{~dB})$} & \multicolumn{2}{|c}{$E_{b} / N_{0}$ at decoder output $(\mathrm{dB})$} \\
\cline { 2 - 3 } & Conventional & Phase diversity \\
\hline 5 & 11.2 & 16.1 \\
6 & 11.8 & 16.7 \\
7 & 12.5 & 17.3 \\
8 & 13 & 17.9 \\
\hline
\end{tabular}

\subsection{Performance of entire access channel decoder}

According to the requirement regarding the access probe test, specified by IS-97D [21], the access failure probability should not excess $1 \%$ when $E_{b} / N_{0}$ is $6.5 \mathrm{~dB}$. In this section, the access failure probability provided by the proposed access channel decoder is presented. Note that the access failure is detected from the failure of CRC contained in the received access probe.

Figures 11 and 12 illustrate the access failure probability in AWGN and fading circumstances, respectively. Note that the required $E_{b} / N_{0}$ for the failure probability to be $1 \%$ is enhanced by about $5 \mathrm{~dB}$. It particularly means that the smart antenna BTS employing the phase diversity can increase the cell size, compared to a conventional BTS consisting of a single antenna channel, so much that the access signal arrives at the BTS with the power of about $5 \mathrm{~dB}$ lower. Consequently, communication performance can be enhanced even during the access state for which the pilot data are not available such that the optimal parameters for nice beam pattern are not yet obtained.

Table 1 represents the measurements of $E_{b} / N_{0}$ at the output port of the proposed access channel decoder of our smart antenna system prepared together with the noise generator and fading emulator as shown in Figure 13. As shown in the table, the proposed access channel decoder enhances the $E_{b} / N_{0}$ by about $5 \mathrm{~dB}$. Note that the measurements shown in Table 1 very much coincides with the simulation results shown in Figures 11 and 12. 


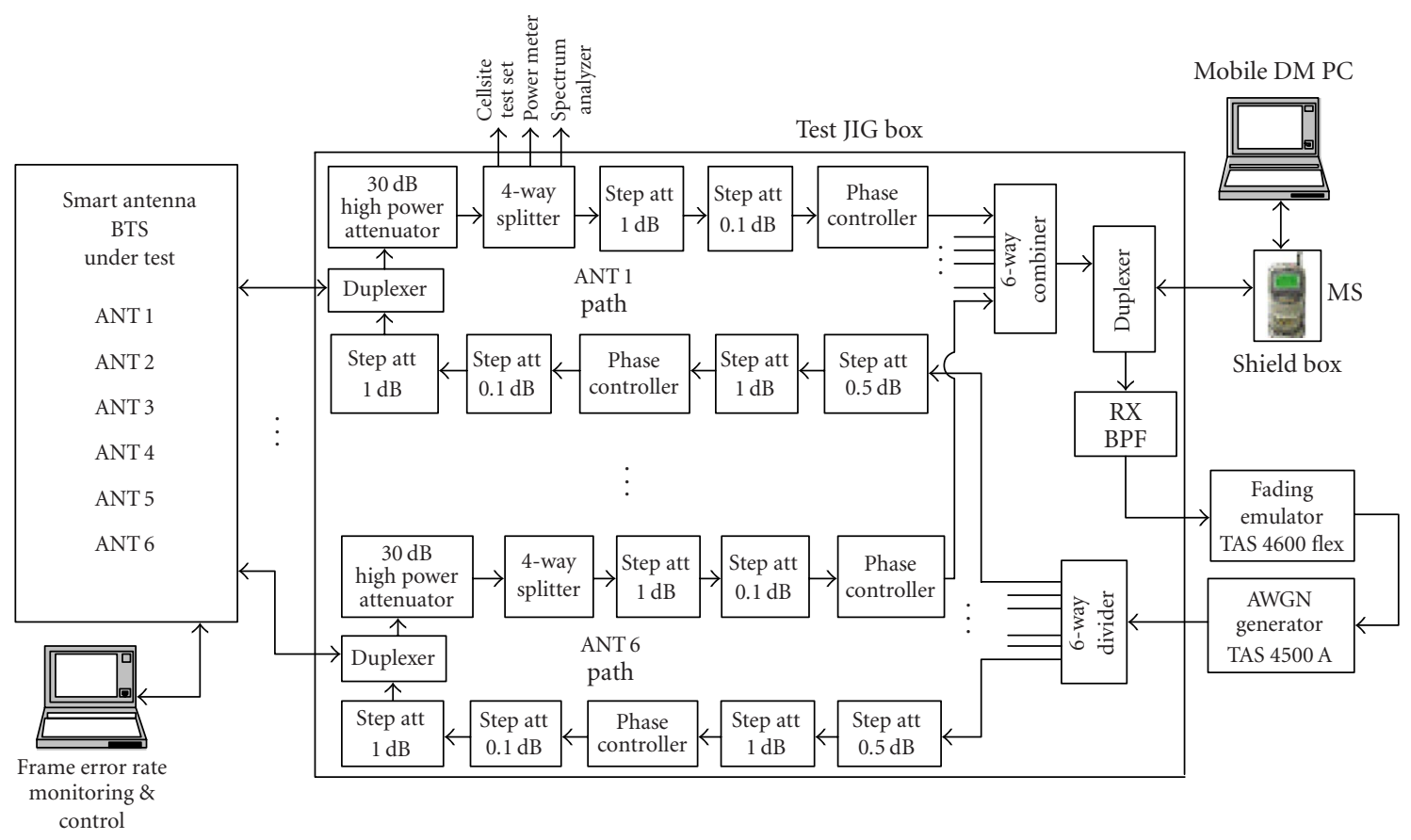

FIGURE 13: Experimental environment for access channel decoder of smart antenna BTS.

\section{CONCLUSIONS}

In this paper, we present an access channel decoder for the smart antenna BTS. The proposed decoder consists of a searcher, OQPSK demodulator, Walsh decoder, Viterbi decoder, and CRC checker. The proposed smart antenna system implemented in this paper exploits the gain of phase diversity with 6 antenna elements. The performance of the proposed access channel decoder has been demonstrated for the access searcher and Walsh demodulator in terms of enhanced detection/false alarm probability and improved $E_{b} / N_{0}$, respectively. The overall performance of the proposed access channel decoder has been presented in terms of access failure probability. As for the access probe test required by IS-97D [21], the proposed access channel decoder provides about $5 \mathrm{~dB}$ improvement in the minimum $E_{b} / N_{0}$ required for the failure rate to be less than $1 \%$ both in AWGN and the fading circumstances. More specifically speaking, for a cell with a radius of $2 \mathrm{~km}$, for example, the proposed technique can increase the distance of reverse search coverage by about $76 \%$ if the path loss in a given mobile environment is proportional to the fourth-power of the path distance [22]. The phase diversity technology proposed in this paper can be used in various systems of next generation mobile communications such as WCDMA systems as well as CDMA2000 1X systems.

\section{ACKNOWLEDGMENT}

This work was supported by HY-SDR Research Center at Hanyang University, Seoul, Korea under the ITRC program of MIC, Korea.

\section{REFERENCES}

[1] F. Adachi, M. Sawahashi, and H. Suda, "Wideband DS-CDMA for next-generation mobile communications systems," IEEE Communications Magazine, vol. 36, no. 9, pp. 56-69, 1998.

[2] A. J. Paulraj and B. C. Ng, "Space-time modems for wireless personal communications," IEEE Personal Communications, vol. 5, no. 1, pp. 36-48, 1998.

[3] J. S. Lee and L. E. Miller, CDMA Systems Engineering Handbook, Artech House, Norwood, Mass, USA, 1998.

[4] J. C. Liberti Jr., and T. S. Rappaport, Smart Antennas for Wireless Communications: IS-95 and Third Generation CDMA Applications, Prentice-Hall, Upper Saddle River, NJ, USA, 1999.

[5] B. Widrow, P. E. Mantey, L. J. Griffiths, and B. B. Goode, "Adaptive antenna systems," Proceedings of the IEEE, vol. 55, no. 12, pp. 2143-2159, 1967.

[6] A. J. Paulraj and C. B. Papadias, "Space-time processing for wireless communications," IEEE Signal Processing Magazine, vol. 14 , no. 6, pp. 49-83, 1997.

[7] B. Suard, A. F. Naguib, G. Xu, and A. J. Paulraj, "Performance analysis of CDMA mobile communication systems using antenna array," in Proc. International Conference on Acoustics, Speech and Signal Processing, vol. IV, pp. 153-156, Minneapolis, Minn, USA, April 1993.

[8] J. S. Thompson, P. M. Grant, and B. Mulgrew, "Smart antenna arrays for CDMA systems," IEEE Personal Communications, vol. 3, no. 5, pp. 16-25, 1996.

[9] T. Cover, R. McEliece, and E. Posner, "Asynchronous multiple-access channel capacity," IEEE Trans. Inform. Theory, vol. 27, no. 4, pp. 409-413, 1981.

[10] F. Khaleghi and M. Kavehrad, "A new correlator receiver architecture for noncoherent optical CDMA networks with bipolar capacity," IEEE Trans. Communications, vol. 44, no. 10, pp. 1335-1339, 1996. 
[11] M. K. Varanasi and A. Russ, "Noncoherent decorrelative detection for nonorthogonal multipulse modulation over the multiuser Gaussian channel," IEEE Trans. Communications, vol. 46, no. 12, pp. 1675-1684, 1998.

[12] H. Hwang, J. Park, Y. Kim, S. Ahn, and S. Choi, "Performance improvement of smart antenna system in terms of searching and tracking capability due to phase diversity technique in IS2000 1X signal environment," in IEEE Antennas and Propagation Society International Symposium, vol. 1, pp. 614-617, San Antonio, Tex, USA, June 2002.

[13] A. Papoulis, Probability, Random Variables, and Stochastic Processes, McGraw-Hill, New York, NY, USA, 1965.

[14] 3GPP2 C.S0002-C, "Physical layer standard for cdma2000 spread spectrum systems," Release C, v1.0, pp. 3-23-3-26, May 2002.

[15] H. Park, B. Kang, S. Bang, and Y. Han, "A maximumlikelihood technique for preamble search in a CDMA reverse link," in 1997 IEEE 47th Vehicular Technology Conference, vol. 3, pp. 1614-1618, Phoenix, Ariz, USA, May 1997.

[16] S. Choi, W. Lee, S. Hyeon, H. Bahk, C. Lee, and J. Kim, "Performance of smart antenna base station implemented for CDMA2000 1X," in MPRG Annual Symposium on Wireless Personal Communications, Blacksburg, Va, USA, June 2003.

[17] S. Choi, H. M. Son, and T. K. Sarkar, "Implementation of a smart antenna system on a general-purpose digital signal processor utilizing a linearized CGM," Digital Signal Processing, vol. 7, no. 2, pp. 105-119, 1997.

[18] Y. Kim, H. Im, J. Park, H. Bahk, J. Kim, and S. Choi, "Implementation of smart antenna base station with a novel searcher and tracker for CDMA2000 1X," in IEEE 8th International Conference on Communication Systems, vol. 1, pp. 394-398, Singapore, November 2002.

[19] A. F. Naguib, Adaptive antennas for CDMA wireless networks, Ph.D. Dissertation, Department of Electrical Engineering, Stanford University, Stanford, Calif, USA, August 1996.

[20] S. Choi, J. Choi, H. Im, and B. Choi, "A novel adaptive beamforming algorithm for antenna array CDMA systems with strong interferers," IEEE Trans. Vehicular Technology, vol. 51, no. 5, pp. 808-816, 2002.

[21] 3GPP2 C.S0010-B, "Recommended minimum performance standards for cdma2000 spread spectrum base stations," Release B, v1.0, pp. 3-23-3-26, December 2002.

[22] J. S. Lee, Lectures on IS-95 CDMA Cellular System Design and the Principles of Cell Planning, J. S. Lee Associates, Rockville, Md, USA, 1996.

Seongdo Kim was born in Daegu, Korea, on March 19, 1965. He received his B.S. and M.S. degrees in electronic communication engineering from Hanyang University, Seoul, Korea, in 1988 and 1990, respectively. He is pursuing the Ph.D. degree in the Department of Electronic Communication Engineering at Hanyang University, Seoul, Korea. From 1990 to 1999, he worked as a Senior Researcher at the Agency for Defence Development in Daejeon, Korea, engaged in the design of military communication system. From 2000 to 2002, he worked as a Senior Researcher at Hyundai electronic in Icheon, Korea, engaged in the design of wideband digital IF and RF subsystem of 3G BTS. In 2003, he joined Ace Technology, Seoul, Korea, where he is presently the Director of Media Communication Institute. His research interests are smart antenna algorithm, design of CDMA BTS system, and application of digital predistortion (DPD) technology.

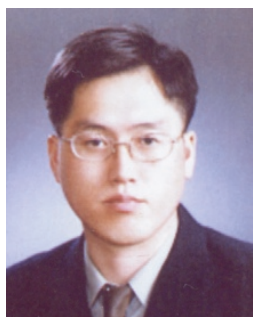

Seungheon Hyeon received his B.S. and M.S. degrees in electronic communication engineering from Hanyang University, Seoul, Korea, in 2002 and 2004, respectively. Since 2002, he has been with the Communication Signal Processing Laboratory, Hanyang University, Seoul, Korea, where he had developed the smart antenna beamforming module and a DSP algorithm for real-time applications. His current research focuses on implementation of a smart antenna system for thirdgeneration mobile communication systems.

Seungwon Choi received his B.S. degree from Hanyang University, Seoul, Korea, and his M.S. degree from Seoul National University, Korea, in 1980 and 1982, respectively, both in electronics engineering, his M.S. degree (computer engineering) in 1985, and his Ph.D. degree (electrical engineering) in 1988, both from Syracuse University, Syracuse, NY. From 1988 to 1989, he was with the Department of Electrical and

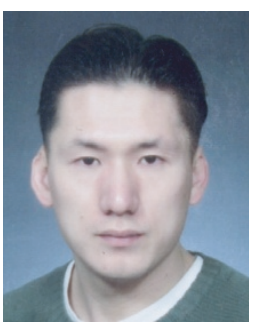
Computer Engineering, Syracuse University, Syracuse, NY, as an Assistant Professor. In 1989, he joined the Electronics and Telecommunications Research Institute, Daejeon, Korea. From 1990 to 1992, he was with the Communications Research Laboratory, Tokyo, Japan, as a Science and Technology Agency Fellow, developing the adaptive antenna array systems and adaptive equalizing filters. He joined Hanyang University, Seoul, Korea, in 1992, as An assistant Professor. He is a Professor at the School of Electrical and Computer Engineering, Hanyang University. Since 2003, Dr. Choi has been serving as the representative of the ITU Region 3 for SDR (Software Defined Radio) Forum. His research interests include digital communications and adaptive signal processing with a recent focus on the implementation of the smart antenna systems for both mobile communication systems and wireless data systems. 